ARTICLE

\title{
Genomic basis of homoploid hybrid speciation within chestnut trees
}

\author{
Yongshuai Sun (1) ${ }^{1,2 凶}$, Zhiqiang $\mathrm{Lu}^{1,2}$, Xingfu Zhu' \& Hui Ma (iD ${ }^{1}$
}

Hybridization can drive speciation. We examine the hypothesis that Castanea henryi var. omeiensis is an evolutionary lineage that originated from hybridization between two nearsympatric diploid taxa, C. henryi var. henryi and C. mollissima. We produce a high-quality genome assembly for mollissima and characterize evolutionary relationships among related chestnut taxa. Our results show that $C$. henryi var. omeiensis has a mosaic genome but has accumulated divergence in all 12 chromosomes. We observe positive correlation between admixture proportions and recombination rates across the genome. Candidate barrier genomic regions, which isolate var. henryi and mollissima, are re-assorted in the hybrid lineage. We further find that the putative barrier segments concentrate in genomic regions with less recombination, suggesting that interaction between natural selection and recombination shapes the evolution of hybrid genomes during hybrid speciation. This study highlights that reassortment of parental barriers is an important mechanism in generating biodiversity.

\footnotetext{
${ }^{1}$ CAS Key Laboratory of Tropical Forest Ecology, Xishuangbanna Tropical Botanical Garden, Chinese Academy of Sciences, Mengla 666303 Yunnan, China.

${ }^{2}$ Center for Plant Ecology, Core Botanical Gardens, Chinese Academy of Sciences, Mengla 666303 Yunnan, China. ${ }^{凶}$ email: sunyongshuai@xtbg.ac.cn
} 
ybridization can generate species without changes in ploidy by reassembling genomes originating from different evolutionary histories into a lineage that is reproductively isolated from the parental lineages ${ }^{1,2}$. This mode of speciation, called homoploid hybrid speciation (HHS), is thought to occur only with difficulty because of, e.g., homogenizing effects from parental lineages ${ }^{2}$. However, theoretical and simulationbased studies suggest that HHS can occur through a reorganization of the preexisting variants which restrict gene exchange between parental lineages or establishing barriers by hybridization-induced chromosomal rearrangements ${ }^{3-5}$. Recently, the reassortment of parental barriers with or without fitness advantages has been considered to be likely as a common mechanism during hybrid speciation ${ }^{6-9}$. Particularly when meeting ecological opportunities, hybrid progenies with recombinant alleles may evolve to become an independent species under the influence of natural selection ${ }^{6}$. Unfortunately, it is still challenging to determine the genes underlying reproductive isolation (RI) between putatively parental species, hindering establishment of the causal link between hybridization and speciation ${ }^{10}$. Large numbers of artificial hybrids/crosses are necessary to locate the loci or genomic regions, which prevent gene flow between parental lineages using traditional approaches ${ }^{7,9}$, such as QTL or genetic mapping ${ }^{11}$. Thus more approaches are needed in order to understand the difference between model expectations and empirical findings for the incidence of HHS. Recent population genomic methods may provide opportunities to address this challenge.

Reduced introgression and enhanced divergence are expected to be exhibited at loci responsible for barriers to gene flow (i.e., barrier loci ${ }^{12}$ ) and the neutral loci linked to such barrier loci, due to selection against unfit hybrids. Genomic regions that are statistical outliers for divergence among populations have been a focus of attention in the recent past, with the aim of identifying barrier loci ${ }^{12}$. However, besides reproductive barriers between species, intraspecific factors (e.g., linked selection and demography) could also influence the genomic landscape of divergence $^{12}$. Another approach for identifying barrier loci is the wellknown genome cline analysis method, which compares locusspecific introgression with the genome-wide average, based on the fact that introgression can vary across the genome due to recombination ${ }^{13}$. A third approach is genomic scanning of linkage disequilibrium (LD) between unlinked genomic regions in hybrids, with the assumption that genetic incompatibilities separating species should show strong patterns of $\mathrm{LD}^{14}$. The above methods appear to be powerful in certain scenarios. However it is still unclear how heterogeneous recombination rates across genome affect these analyses. Here, we assumed that most genomic regions with mixed genetic compositions are likely to exert no/weak isolating effects and used a sophisticated experimental design and advanced approaches to capture historical strengthening of barrier effects and identify barrier genomic regions among chestnut lineages.

Chinese chestnut trees are thought to be critical for resurrecting wild American chestnut and European chestnut trees, which are being eliminated by Cryphonectria parasitica ${ }^{15-18}$. Knowledge of speciation genomics may be of great value to the chestnut forest restoration in Europe and America. We used phylogenetic control, the exclusiveness of barrier alleles in the hybrid lineage and backward simulations to identify/test candidate genomic regions responsible for RI between Castanea mollissima Blume $\mathrm{e}^{19}$ and variety henryi of Castanea henryi (Skan) Rehder \& E. H. Wilson. The candidate RI genomic regions would provide information for testing the role of hybridization in the origin of C. henryi var. omeiensis, which was discovered in the west of China (on Mount Emei) in 1964. Initially, it was suspected to be a hybrid lineage or hybrid (C. mollissima $\times$ C. henryi var. henryi). Morphologically, C. mollissima trees are characterized by three nuts per bur, differing from $C$. henryi trees which have one nut per bur ${ }^{19}$. However, the leaves of $C$. henryi var. omeiensis are covered with stellate tomentose hairs abaxially ${ }^{19}$, and morphologically similar to those of C. mollissima, providing inconclusive evidence for a hybrid origin hypothesis. Geographically ${ }^{20}, C$. henryi, endemic in China, appears to be broadly sympatric across most of the range of C. mollissima (Fig. 1a). The shrub-like species Castanea seguinii, characterized by glabrous leaves and three nuts per bur, also has a similar distributional range to $C$. mollissima and $C$. henryi. In addition, the flowering periods of $C$. mollissima, C. seguinii and C. henryi have overlap ${ }^{19}$, although some C. mollissima trees begin to flower in late April. Their distributions and flowering periods provide sufficient opportunities for interbreeding. Artificial hybridization experiments with C. mollissima and C. henryi showed that the seeding rate and empty-bur rate resulting from interbreeding were higher than those following normal pollination ${ }^{21}$. These interbreeding experiments and the distinct phenotypes of C. mollissima and $C$. henryi, together with their distributions, are suggestive of the existence of reproductive barriers typically isolating C. mollissima from C. henryi. A phylogenetic tree of all Castanea species suggested that these four taxa, which are endemic in China, constitute a monophyletic group, sister to the clade containing the European chestnut and the American chestnut ${ }^{22}$. Genetic variation within species indicated that $C$. seguinii and C. mollissima likely constituted a single clade although past hybridization might have occurred among these four diploid chestnut taxa ${ }^{23}$.

Here, we test the hypothesis that $C$. henryi var. omeiensis originated from hybridization between $C$. henryi var. henryi and $C$. mollissima, using multiple population genomic approaches and simulation-based tests. We identify candidate barrier genomic regions between $C$. henryi var. henryi and C. mollissima, based on genomic variation and a de novo assembled reference genome generated from Nanopore sequencing data ${ }^{24}$ and highthroughput chromosome conformation capture (Hi-C) ${ }^{25}$ short reads. We find that $C$. henryi var. omeiensis has a distinct genetic structure, and that both of the parental lineages contributed substantial genomic material to the hybrid lineage, a phenomenon referred to as genomic mosaicism ${ }^{10}$. Next, taking advantage of the phylogenetic control in this system, we identify the candidate barrier genomic regions by seeking genomic regions with reduced gene flow between parental lineages and filtering regions with mixed alleles from different species. Finally, we examine the mosaic of barrier genomic regions within the putatively hybrid lineage and investigate their distribution across chromosomes.

\section{Results}

Genome assembly. We chose one C. mollissima tree to perform single-molecule Nanopore DNA sequencing, and construct a Hi$C$ library for scaffolding after assembling reads into contigs; we selected this species because C. mollissima trees are cultivated widely in China and thus may have lower heterozygosity relative to the other three taxa (C. seguinii, $C$. henryi var. henryi and var. omeiensis). The haploid genome size of C. mollissima was measured by flow cytometry as 773-785 megabase pairs (Mbp), consistent with a previous report ${ }^{26}$. The Illumina HiSeq platform produced short reads with a total size of 80.8 billion base pairs (Gbp). The $k$-mer frequency analysis $(k=17)$ using Jellyfish ${ }^{27}$ reported a smaller genome size, of $706.3 \mathrm{Mb}$, relative to precious reports ${ }^{26}$. A total of $100.6 \mathrm{Gbp}(\sim 128 \times$ coverage $)$ of Nanopore sequence data were used, after filtration, in the assembly analysis which produced 422 contigs with an $N_{50}$ of $5.88 \mathrm{Mbp}$ and total length of $773.99 \mathrm{Mbp}$ (Supplementary Table 1). The longest 

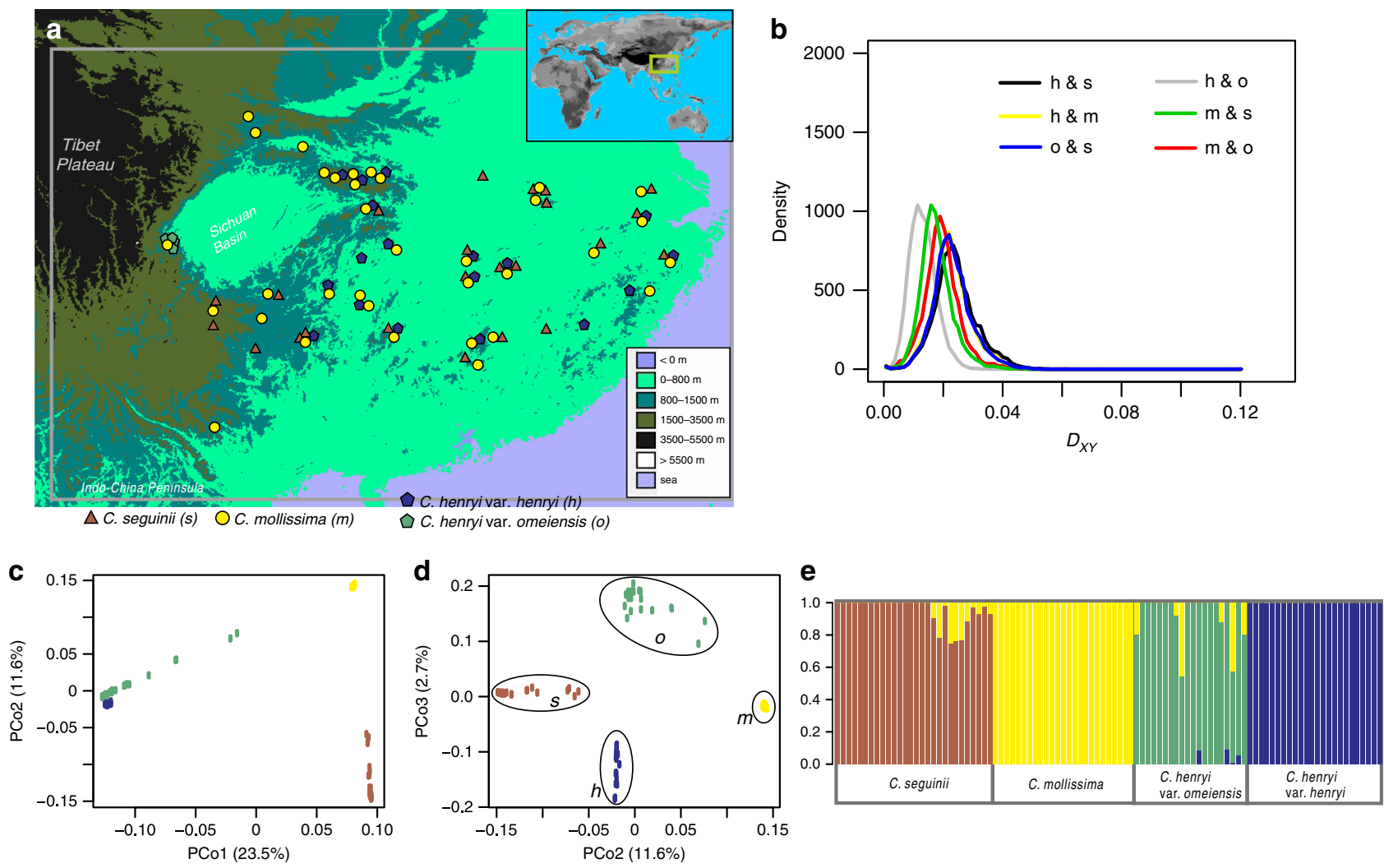

f

$\max F_{\mathrm{ST}}$ between $h$ and $o$

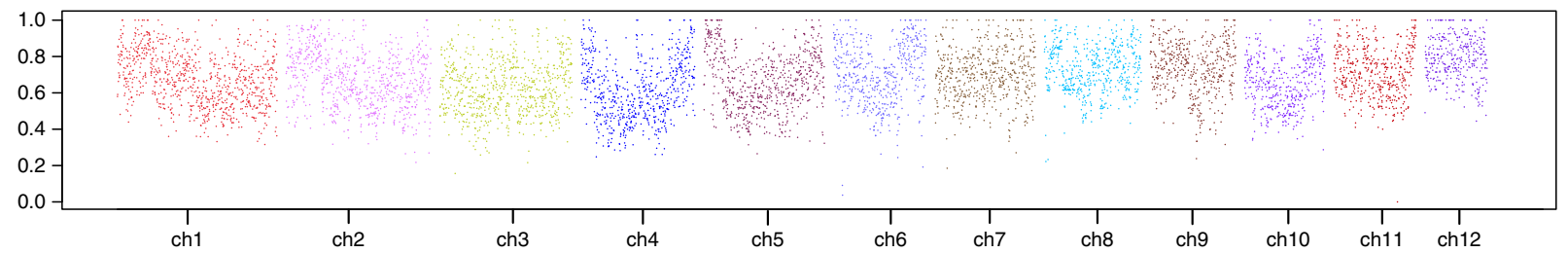

g

$\max F_{\mathrm{ST}}$ between $m$ and $o$

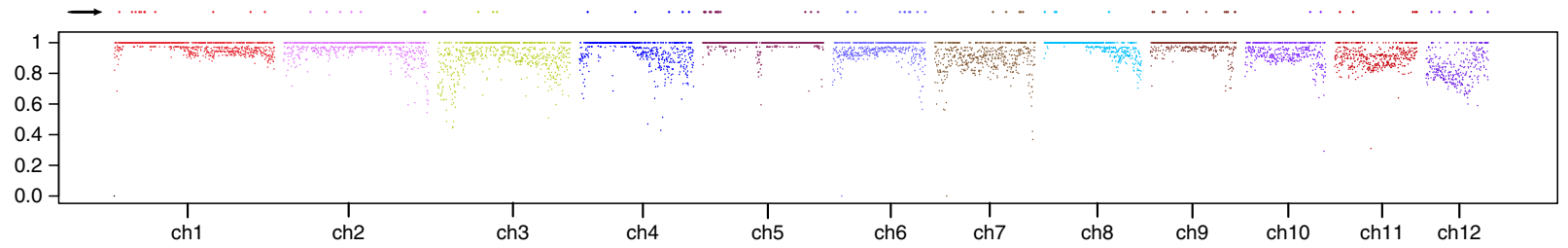

Fig. 1 The four Castanea taxa show distinct genomic variation. a Map showing the distributions of samples collected. Each of the four taxa is shown in a different color. $\mathbf{b}$ Frequency polygon of $D_{\mathrm{XY}}$ per base pair for each pair out of the four taxa, using a sliding-window approach with a window size of $100 \mathrm{kbp}$. Abbreviations are: $\mathrm{s}=\mathrm{C}$. seguinii, $\mathrm{m}=\mathrm{C}$. mollissima, $\mathrm{h}=\mathrm{C}$. henryi var. henryi, $\mathrm{o}=\mathrm{C}$. henryi var. omeiensis (the same below). $\mathbf{c}, \mathbf{d} \mathrm{PCoA}$ showing the first three coordinates and the percentage of variation explained by each coordinate. The four colors represent samples collected from the four taxa. e Population structure estimated by admixture analysis for all four Castanea taxa. $\mathbf{f} \max _{\mathrm{ST}}$ analysis of $100 \mathrm{kbp}$ nonoverlapping windows across the 12 chromosomes between $C$. henryi var. henryi and C. henryi var. omeiensis. The 12 chromosomes are represented by different colors. $\mathbf{g}$ max $F_{\mathrm{ST}}$ analysis of $100-\mathrm{kb}$ nonoverlapping windows across the 12 chromosomes between $\mathrm{C}$. mollissima and $\mathrm{C}$. henryi var. omeiensis. The arrow in $\mathbf{g}$ indicates windows where fixed differences between $C$. henryi var. omeiensis and the two parental taxa ( $C$. henryi var. henryi and $C$. mollissima) can be observed. Source data underlying Fig. $1 b-g$ are provided as a Source data file.

contig was $41.15 \mathrm{Mb}$. We used Illumina short reads to polish these assembled contigs. Most of the Illumina short reads (98.45\%) could be aligned to these 422 contigs using BWAMEM $^{28}$. We performed BUSCO V3.0.129 assessment, and $97.4 \%$ of the core genes were found to be complete in the assembled genome. This assembly appears to be better than the previous version $^{26,30}$, despite the smaller total genome size (Supplementary Table 1). Chromosome-scale scaffolding analysis based on $\mathrm{Hi}-\mathrm{C}$ data assembled 377 contigs into 12 pseudo-chromosomes with a total length of $734.13 \mathrm{Mbp}$ (Supplementary Fig. 1). More than half of the C. mollissima genome (59\%) was identified as repetitive elements and $31.70 \%$ of the genome as retroelements (Supplementary Tables 2 and 3). $33.69 \%$ and $50.82 \%$ of the retroelements were classified as Copia and Gypsy, respectively. A total of 45,661 gene models were predicted, of which 45011 were protein coding genes (Supplementary Tables 4 and 5). This is 
considerably more than the 30832 gene models reported in the previous study ${ }^{30}$, needing further experimental confirmation.

Population re-sequencing. We carried out whole-genome resequencing of 20 trees of $C$. henryi var. omeiensis, $24 C$. henryi var. henryi trees, 43 C. mollissima trees and 28 C. seguinii trees, spanning their geographic ranges (Fig. 1; Supplementary Tables 6-9). We generated approximately $25.7 \times$ sequence coverage per individual tree by sequencing $2 \times 150 \mathrm{bp}$ paired-end reads. Reads were mapped on the high-quality de novo assembled reference genome created for C. mollissima. Then we identified heterozygous and homozygous genotype per base for each individual tree using a variant-calling pipeline and GATK $4^{31}$. We validated the quality of the variant-calling procedure based on duplicated re-sequencing of seven trees. The maximum proportion of unverified heterozygotic genotypes for the seven trees was $0.000167 \%$, corresponding to a Phred-scaled score of 57.8 , suggesting that variants detected by our GATK4-pipeline were of high quality. The putative hybrid taxon $C$. henryi var. omeiensis had the highest observed heterozygosity $(1.05 \pm 0.26 \%)$, when compared to C. mollissima $(0.66 \pm 0.05 \%)$, C. henryi var. henryi $(0.85 \pm 0.08 \%)$ or C. seguinii $(0.88 \pm 0.11 \%)$. Sliding-window analysis with a size of 100 kilobase pairs $(\mathrm{kbp})$ for each of the four taxa suggested that (Supplementary Fig. 2) C. mollissima had the lowest nucleotide diversity $(\pi=0.0075 \pm 0.0039)$, compared to C. henryi (for var. henryi, $0.0108 \pm 0.0045$; for var. omeiensis, $0.0108 \pm 0.0037)$ and $C$. seguinii $(0.0100 \pm 0.0043)$. The slidingwindow analysis of mean nucleotide differences between lineages ( $D_{\mathrm{XY}} ; 100 \mathrm{kbp}$ ) showed that the $D_{\mathrm{XY}}$ between C. mollissima and $C$. seguinii was less than the $D_{\mathrm{XY}}$ between $C$. henryi varieties (Fig. 1b), suggesting that divergence between C. mollissima and $C$. seguinii likely predated the origin of $C$. henryi var. omeiensis.

Distinct genetic structure and fixed differences. Genetic analyses of genome-wide SNPs suggest that C. henryi var. omeiensis is an independent evolutionary lineage. First, a principal coordinate analysis (PCoA) indicated an intermediate position for C. henryi var. omeiensis along the second principal coordinate (PCoA2), but a distinct position for $C$. henryi var. omeiensis along PCoA3 (Fig. 1c, d). PCoA results were mirrored by the results of individual-based clustering analyses using ADMIXTURE ${ }^{32}$ and FASTSTRUCTURE ${ }^{33}$. For these four taxa, which had been identified based on morphological taxonomy, the best-fit model supported four distinct populations, albeit with substantial admixture among taxa (Fig. 1e). Eleven trees of C. seguinii seemed to be recent hybrids or backcrosses resulting from hybridization between $C$. seguinii and C. mollissima. With a setting of $K=5$, FASTSTRUCTURE could not detect any extra distinct cluster. Finally, we used sliding-window analysis of fixation index $\left(F_{\mathrm{ST}}\right.$, $100 \mathrm{kbp}$ ) to assess population differentiation between $C$. henryi var. omeiensis and its putative parental taxa, setting sample size per taxon to be 20. To investigate the fixed nucleotide difference, we used $\max F_{\mathrm{ST}}$, which represented the maximum $F_{\mathrm{ST}}$ per bp across one window. Consistent with observations of morphology $^{19}$, windows with $\max F_{\mathrm{ST}}=1.0$ between $C$. henryi var. henryi and var. omeiensis were far fewer than those between $C$. henryi var. omeiensis and C. mollissima (Fig. 1f, g). Furthermore, this sliding-windows determination of $\operatorname{maxF}_{\mathrm{ST}}$ showed that fixed differences between $C$. henryi var. omeiensis and the putative parental taxa could be found in any of the 12 chromosomes, especially at the chromosome peripheries. The high level of interspecific differentiation suggested that some reproductive barriers may have segregated $C$. henryi var. omeiensis from its parental lineages.
Mosaic genomes. To investigate the genomic mosaicism, we introduced a statistic $h h s=d_{\mathrm{om}} / d_{\mathrm{hm}} \times 100 \%$, where $d_{\mathrm{om}}$ and $d_{\mathrm{hm}}$ represented mean nucleotide differences between $C$. mollissima and the two $C$. henryi varieties. If $C$. mollissima contributed some genetic components to the genome of $C$. henryi var. omeiensis, the statistic $h$ hs would be $<100 \%$. The $h$ hs estimated by our slidingwindow (100 kbp) analysis was $86 \%$ on average and a bootstrap test significantly $(P<0.001)$ refuted the hypothesis of $h h s=100 \%$. In concordance with this, coalescent modeling of the genomewide interspecific site frequency spectrum (2D-SFS) generated by ANGSD $^{34}$ clearly favored a hybrid speciation model leading to the formation of $C$. henryi var. omeiensis over models in which $C$. henryi var. omeiensis was sister to either C. henryi var. henryi or C. mollissima (Supplementary Fig. 3). In the hybrid speciation model, the $H$ parameter represented the relative proportion of the genetic composition derived from C. mollissima (Fig. 2a). The estimated $H$ was $24.4 \pm 1.5 \%$, indicative of $C$. mollissima being the minor parental taxon. To investigate in detail the parental contributions along each of the chromosomes, we split the genome into nonoverlapping windows (1 Mbp; Fig. 2a). We used HyDe ${ }^{35}$ to compute a point estimator of $H$ for each individual chestnut tree. The estimated $H$ values varied across all $C$. henryi var. omeiensis individuals in a range from 11.87 to $44.32 \%$ (Fig. 2b), and they also varied across the assembled chromosomes (Fig. 2c). These results together with those of the ADMIXTURE and PCoA analyses provided substantial evidence for genomic mosaicism in this system.

Design and rationale for testing HHS. The approaches used to test for signals of hybridization by analyzing the ABBA, BABA, and BBAA polymorphic patterns in a four-taxon scenario (e.g., $\mathrm{HyDe}^{35}$, Supplementary Fig. 4$)$, which we set as ((s, m), h, outgroup), have been found to be powerful and computationally efficient on a genomic scale ${ }^{36,37}$. These approaches take advantage of phylogenetic control to distinguish incomplete lineage sorting from hybridization between $\mathrm{h}$ and $\mathrm{m}$ (or s). Here, we used the HyDe ${ }^{35}$ method to identify the barrier genomic regions isolating C. henryi var. henryi and C. mollissima. In our approach, we specify $A_{s m}$ to represent the ancestral lineage of $m$ and $s$ (Supplementary Fig. 4). At a locus (L) arising from hybridization between $h$ and $s$, gene exchange can occur between $h$ and $A_{s m}$ at the L locus, unless extrinsic barriers (such as geographic barriers) hinder hybridization between them. Following divergence between s and $\mathrm{m}$, some barrier loci, which impede gene exchange between $h$ and $m$ but allow migration between $h$ and $s$, can evolve from loci that were not previously barriers between $h$ and $A_{s m}$. One major signature of such barrier loci is that gene flow can be found between $h$ and $s$ but not between $h$ and $m$. When hybridization occurs, mixed barrier alleles sourced from different species can cause maladaptation, due to deleterious effects of mixed variation at one or more barrier loci ${ }^{38-41}$. If hybrid speciation could occur, barrier alleles from one parental population would evolve to be fixed in hybrids (or to be replaced completely by alleles from the other parent in subsequent generations), due to the purging effect of natural selection ${ }^{6,42}$. These two properties thus appear to provide means of identifying barrier genomic regions.

Mosaic of barrier genomic regions. We examined the correlation between recombination rates $(\rho)$ and admixture proportions (using $H$ ). Several models of divergence under gene flow predicted positive correlations between recombination rates and admixture proportions when multiple barrier loci were involved $^{43}$. We generated a LD-based recombination map for $C$. mollissima using LDhelmet $^{44}$, taking into account specific 
a

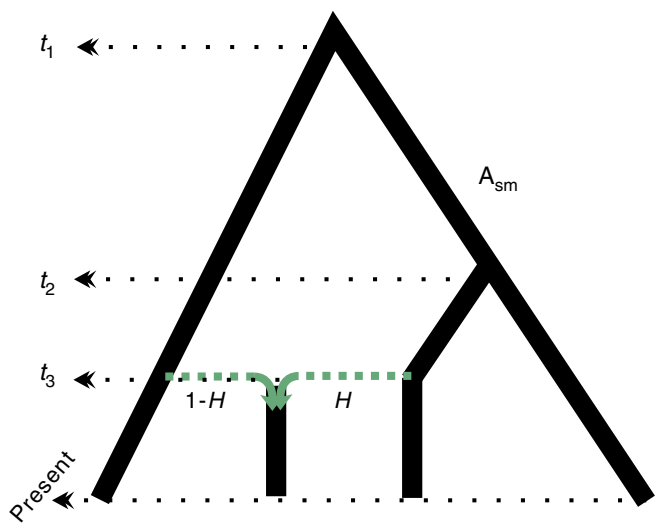

b
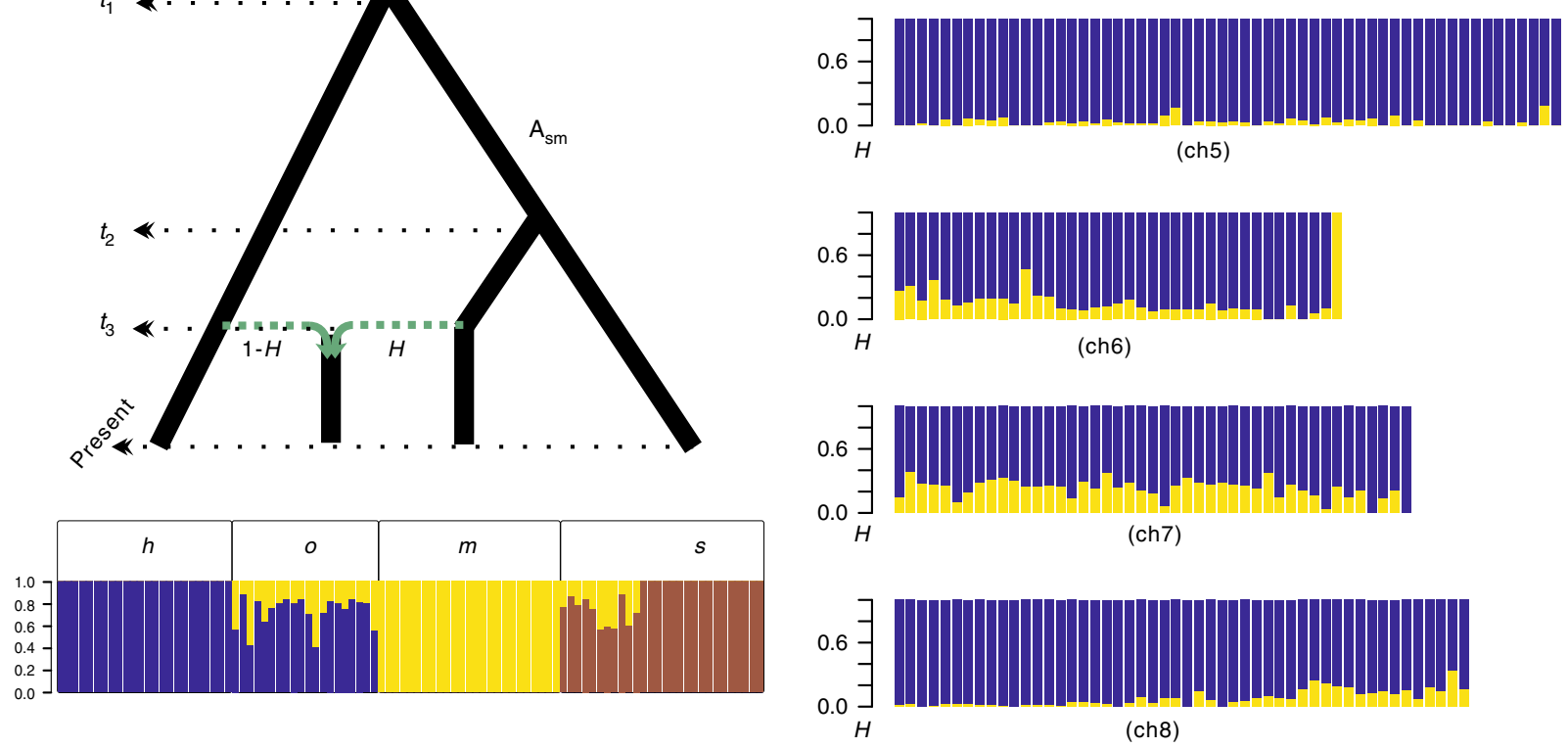

\section{C}
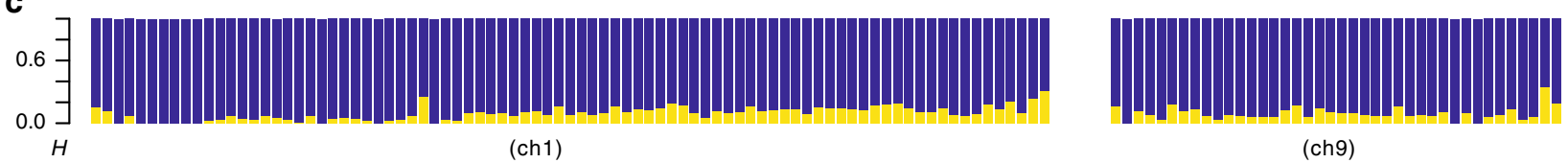

(ch9)
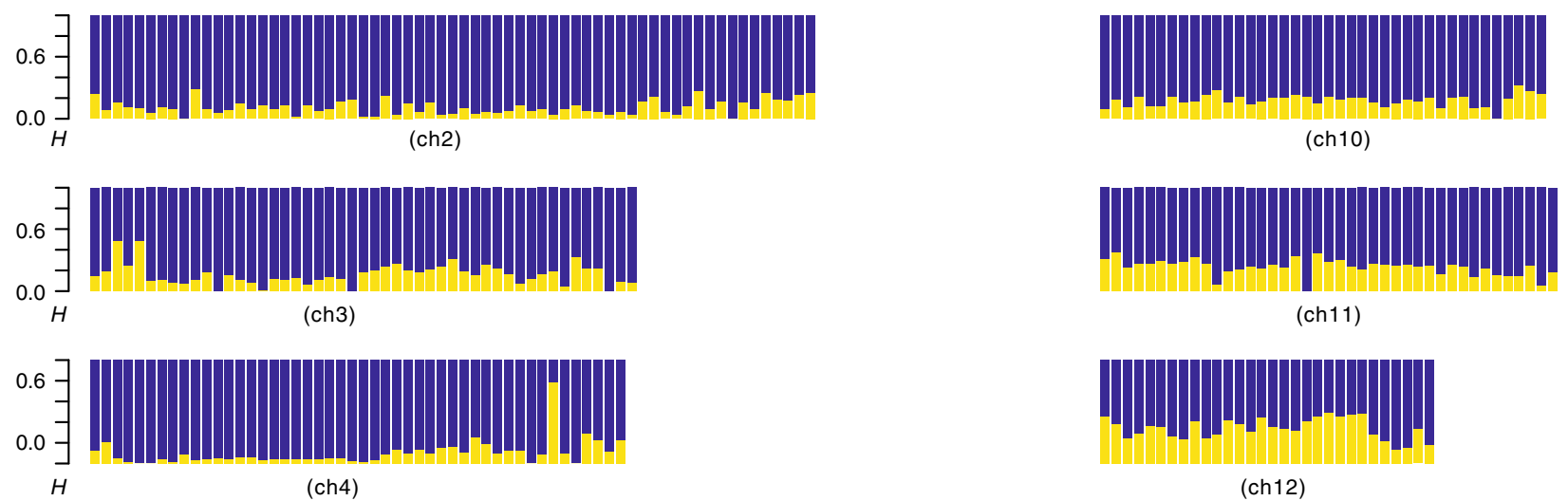

Fig. 2 Genomic mosaicism. a The most likely evolutionary model used in the present study. At time $t_{1}$, the lineage $h$ diverged from the ancestral lineage

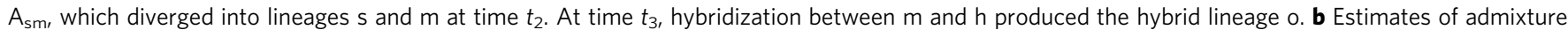
proportions for each individual tree. c Estimated $H$ for 100-kbp nonoverlapping windows across the 12 chromosomes of $\mathrm{C}$. henryi var. omeiensis, from ch1 to ch12. Source data underlying $(\mathbf{b}, \mathbf{c})$ are provided as a Source Data file.

mutation matrices and the effects of recent changes in population size $^{45}$ (Supplementary Table 10; Supplementary Figs. 5 and 6). The $H$ values estimated for genomic regions of $C$. henryi var. omeiensis increased with the local recombination rates (Fig. 3a), consistent with the hypothesis that the species barriers between $C$. mollissima and $C$. henryi var. henryi are highly polygenic ${ }^{46}$. In addition, we calculated the correlation for the 11 possible hybrids that may result from hybridization between $C$. mollissima and $C$. seguinii (Figs. 1e and $2 \mathrm{~b}$ ), taking these putative hybrids as a single group. The same pattern could also be observed for these hybrids (Fig. 3b), indicating that polygenic barriers might be common in chestnut species.

Using C. seguinii as an ingroup, we identified the genomic regions (window size $=100 \mathrm{kbp}$ ) with significant introgression between $C$. henryi var. henryi and C. mollissima (denoted $M_{\mathrm{hm}}$ ), using $\mathrm{HyDe}^{35}$. Using C. mollissima as an ingroup, we identified the genomic regions with significant introgression between $C$. henryi var. henryi and C. seguinii (denoted $M_{\mathrm{hs}}$ ). For loci in $M_{\mathrm{hs}}$, gene exchange at these loci could occur between $C$. henryi and the ancestor of $C$. mollissima and $C$. seguinii. During the divergence of C. mollissima and C. seguinii, the barrier genomic regions, which could prevent gene flow between $C$. henryi and $C$. mollissima would fall into $M_{\mathrm{hs}}$ but not $M_{\mathrm{hm}}$. Migration between C. henryi and the ancestor of C. mollissima and C. seguinii at the genomic regions in $M_{\mathrm{hs}}$ but not in $M_{\mathrm{hm}}$ would have been reduced when C. mollissima and C. seguinii diverged (Supplementary Fig. 7). Thus genomic regions in $M_{\mathrm{hs}}$ but not in $M_{\mathrm{hm}}$ might contain genes preventing gene flow between the two parental lineages.

Local ancestry inference for each genomic region in $M_{\mathrm{hs}}$ but not in $M_{\mathrm{hm}}$ suggested that 82 regions in the $C$. henryi var. omeiensis genomes were from C. henryi var. henryi (red rhombus in Fig. 3c), and six from C. mollissima (blue rhombus in Fig. 3c). Two additional results further supported this result. First, fixed 
a

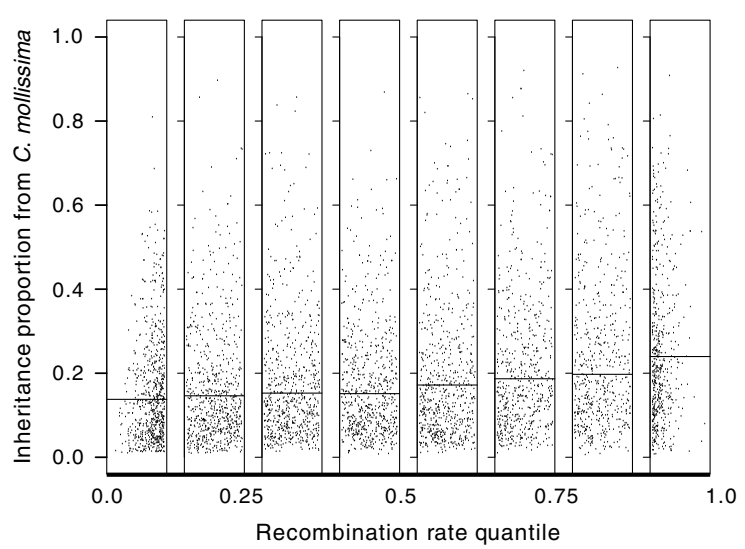

b

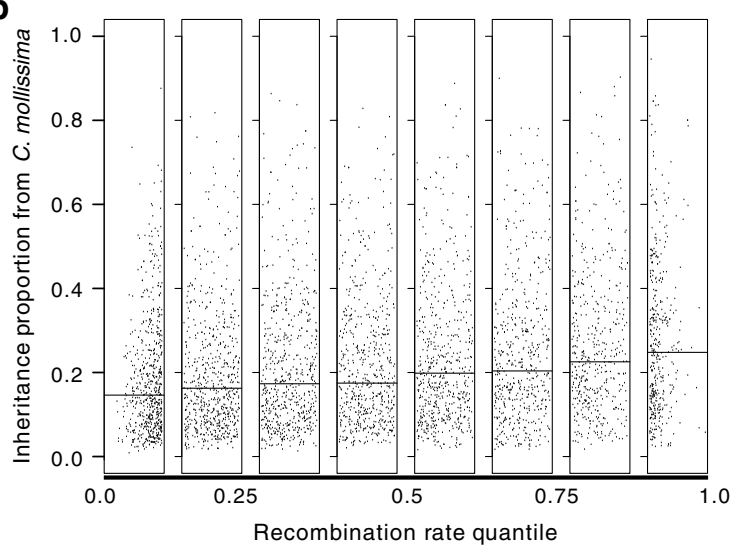

C

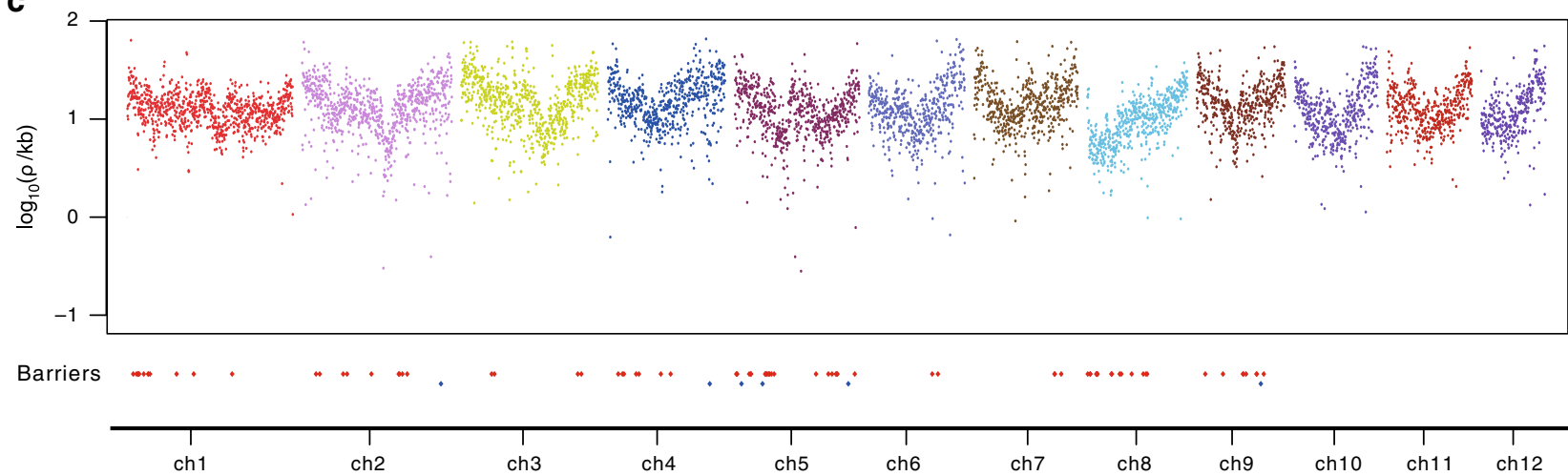

Fig. 3 Mosaic of polygenic barriers. a Relationship between recombination rates and the proportions of $C$. henryi var. omeiensis genetic material inherited from C. mollissima. $\mathbf{b}$ Relationship between recombination rates and the genomic proportions in hybrids (C. mollissima $\times$ C. seguinii) inherited from $C$. mollissima. c Manhattan plots of recombination rates across the 12 chromosomes in the C. mollissima genome (100-kb nonoverlapping window). To illustrate the distribution of candidate barrier genomic regions, these are listed regions below the Manhattan plots of recombination rates. The red rhombi represent candidate barrier genomic regions derived from $C$. henryi var. henryi and the blue rhombi represent regions from $C$. mollissima. Source data are provided as a Source data file.

differences between the two parental lineages could be found in each of 88 candidate barrier regions $\left(\max F_{\mathrm{ST}}=1.0\right)$. Second, coalescent modeling of 2D-SFS generated from these 88 candidate barrier genome regions suggested that gene flow between parental lineages had reduced after the divergence of $C$. mollissima and $C$. seguinii (Supplementary Table 11). Our results revealed that both C. mollissima and C. henryi var. henryi contributed genomic regions preventing parental introgression to $C$. henryi var. omeiensis, therefore directly supporting the HHS hypothesis.

Genomic distribution of barrier regions. Our analysis of the 82 barrier windows from $C$. henryi var. henryi revealed that the number of barrier regions decreased with increasing recombination rate $(F$-statistic $=11.85, P<0.00059)$, implying that barrier loci may tend to concentrate in genomic regions with low recombination rates. The concentration of barrier loci may render an increase in the density of selection against maladaptive immigrant alleles in regions with low recombination rates and may further lead to the correlation between $H$ and recombination rates (Fig. 3a).

Annotation of barrier regions. In candidate barrier genomic regions, 466 protein coding genes were found and two of them (bl_011844 on chromosome c2; bl_014924 on chromosome c8) could be aligned to flowering-time genes in the FLOR-ID database ${ }^{47}$. The bl_011844 gene was aligned to the SIZ1 gene, whose product acts as a floral repressor that can repress the SAdependent pathway and promote FLC expression by repressing FLD activity through sumoylation ${ }^{47,48}$. The $b l \_014924$ gene was aligned to JMJ14, whose product can inhibit the floral transition by repressing the floral integrators $F T, A P 1, S O C 1$ and $L F Y$ through histone $\mathrm{H} 3 \mathrm{~K} 4$ demethylation ${ }^{47,49}$.

\section{Discussion}

The present study produced strong genomic evidence for the hypothesis that $C$. henryi var. omeiensis originated from hybridization between C. mollissima and C. henryi var. henryi, providing an opportunity to search for potential correlations between candidate barrier genomic regions and traits responsible for RI. The genomic variation identified in C. henryi var. omeiensis supported its independent evolutionary status (Fig. 1). The simulation-based test and HyDe analysis of population genomic variation showed that $C$. mollissima and $C$. henryi var. henryi contributed substantial material to the gene pool of $C$. henryi var. omeiensis (Fig. 2), meeting the genomic mosaicism criteria for $\mathrm{HHS}^{10}$. Moreover, we identified some candidate barrier genomic regions (Fig. 3), supporting a polygenic barrier model. Barrier effects in candidate regions separating C. mollissima and C. henryi var. henryi would continue to exclude unfit mixed alleles from $C$. henryi var. omeiensis. That is, the hybrid lineage has inherited preexisting genetic barriers from its two parents, supporting the HHS hypothesis for C. henryi var. omeiensis. In particular, our 
analyses captured the reduction of migration in candidate barrier genomic regions by utilizing phylogenetic control. Our study highlights the importance of using phylogenetic information in identifying molecular segments involved in RI.

When divergence between $C$. seguinii and C. mollissima occurred, the genomic landscape of introgression between either species and $C$. henryi would have evolved independently, due to C. seguinii and C. mollissima beginning to accumulate barrier alleles separately. Depending on the changes of allele or genotype frequencies in hybrids and backcrosses, genome cline analysis and an LD-based genome scan of derived alleles were used to identify candidate barrier loci based on genomic variation in systems without an evolutionary lineage derived from hybridization ${ }^{8,14}$. One extreme scenario is that hybrids fixed genomic regions from one parent, and these might be likely to show the steepest changes in frequency and the strongest LD pattern. It should be noted that frequency changes could also be explained by random drift. To exclude the effects of drift, we employed phylogenetic control and examined the process of reduction in gene flow between parental lineages, with introgression between C. seguinii and C. henryi var. henryi as a background reference. In concordance with morphology ${ }^{19}$, we found that $C$. henryi var. henryi contributed more genetic information to the hybrid lineage in candidate barrier genomic regions $(82 / 88)$. The genes underlying key traits contributing to hybrid speciation were often found to be sourced from different parents in previously reported homoploid hybrid systems ${ }^{10}$, indicating a role for a combinatorial mechanism in initiating species diversification ${ }^{7-9,42,50}$. Consistent with this, our results indicate a role for the assortment of parental barriers in promoting hybrid speciation ${ }^{6}$.

Artificial interbreeding experiments suggested that pre-zygotic barriers may have prevented the incidence of $C$. henryi $\times C$. mollissima hybrids ${ }^{21}$. In support of this, our admixture analysis found no obvious hybridization between $C$. henryi var. henryi and C. mollissima samples (Fig. 1e). The slight difference in flowering periods of C. henryi and C. mollissima ${ }^{19}$, might indicate partial pre-pollination isolation. Genes inherited from $C$. henryi var. henryi may determine the flowering time of the hybrid lineage, because $C$. henryi var. henryi contributed more $(\sim 75.6 \%)$ genetic information to the hybrid taxon. We annotated the candidate barrier genomic regions from C. henryi var. henryi, and found that two genes were closely related to genes (SIZ1 and JMJ14) controlling the flowering-time trait in Arabidopsis plants ${ }^{47-49}$. One focus of future work will be to determine whether there is a direct link between these genes and the difference in flowering time in the two parental taxa. The procedure employed to identify genetic barriers in this study consisted of utilizing the exclusiveness of barrier alleles. Thus, some of the intrinsic genetic incompatibilities, which could further reduce the fitness of hybrids and may have prevented immigrations from parental lineages into the hybrid lineage, may be in the candidate barrier genomic regions. Our results, together with previous studies on chestnut reproduction and phenology ${ }^{19,21}$, suggested that both pre- and post-pollination factors may have contributed to the isolation between $C$. henryi and C. mollissima. The relative importance of chromosomal variation, spatial and ecological factors in speciation also still need comprehensive assessment.

The candidate barrier genomic regions are distributed in nine pseudo-chromosomes (Fig. 3c), separated by at least $0.2 \mathrm{Mbp}$ in all cases. This result indicates that the effective migration rates in these candidate regions would be lower than rates in other genomic regions and chromosomes, revealing a heterogeneous landscape of hybrid ancestry across the genome of the hybrid lineage. Recent works have suggested that natural selection together with recombination could influence the genomic distribution of introgressed alleles from the minor parental species $^{38,51}$, by purging the more deleterious effects of mixed genotypes sourced from different species. The positive correlation between admixture proportions $(H)$ and recombination rates across the genome in our results supports this prediction (Fig. 3a, b). In contrast to previous reports, our analysis reveals that $C$. henryi var. omeiensis has fixed many barrier alleles derived from the major parent $C$. henryi var. henryi in genomic regions with low recombination rates. This result suggests that barrier loci from parental lineages would not be uniformly distributed in the genome of the hybrid lineage, tending rather to be densely distributed in genomic regions with less recombination. The correlation between $H$ and recombination rates and the landscape of hybrid ancestry across the genome (Fig. 3a) may be shaped by the genomic architecture of reproductive barriers ${ }^{40,52}$. Furthermore, the concentration of barrier regions provides an alternative explanation for the high levels of divergence and differentiation in genomic regions with less recombination.

Due to selection against maladaptive mixed barrier genotypes, alleles present at higher frequencies at barrier loci would be favored by natural selection until fixation occurred. Barrier alleles from the major parent (C. henryi var. henryi) may be coupled in the regions of hybrid genomes with less recombination, and thus impede the incorporation of barrier alleles from the minor parent (C. mollissima). This coupling may also have impeded the maintenance of barriers from the minor parent, with the result that C. mollissima contributed far fewer barrier genomic regions (6/88) compared to the genomic composition contributed by $C$. mollissima $(H=24.4 \pm 1.5 \%)$. Furthermore, the six barrier genomic regions sourced from C. mollissima should have prevented gene flow from the major parent (C. henryi var. henryi) to the hybrid lineage, due to the maladaptation of mixed genotypes at barrier loci. Interaction between selection and recombination may therefore have shaped the genomic distribution of parental genetic barriers in the process of forming hybrid species. In future, more studies may be needed in order to assess the relative barrier effect of each candidate locus in this unique hybrid system, and more work in other systems is needed to assess the generality of biased distribution of parental barriers in the genomes of hybrid taxa.

\section{Methods}

Reference genome assembly. DNA from the Chinese chestnut chosen for the reference genome assembly was extracted and used to construct libraries for Nanopore, Illumina and $\mathrm{Hi}-\mathrm{C}$ sequencing. We assembled the Nanopore long reads into contigs and used the Illumina short reads to polish the assembled contigs after filtration (see Supplementary note 1). Then we anchored the contigs based on the $\mathrm{Hi}-\mathrm{C}$ dataset (see Supplementary note 2).

Gene prediction and functional annotation. We used the ab initio and homologybased methods to perform gene model predictions which were used to generate a set of consensus gene models (see Supplementary Notes 3 and 4). The final gene set was functionally annotated using BLASTP with an $E$-value cutoff of $1 \mathrm{e}-5$ against the SwissProt and TrEMBL databases. Homologous protein domains were searched for using InterProScan ${ }^{53}$. The GO terms for hits in the above sequence and domain searches were then assigned to the corresponding C. mollissima genes. KEGG pathway annotation was conducted using $\mathrm{KAAS}^{54}$.

Population samples and resequencing. We collected young leaves from the four taxa spanning their geographic ranges (Fig. 1 and Supplementary Tables 6-9). We filtered out populations $<5 \mathrm{~km}$ distant from villages, cities and man-made chestnut forests, with the aim of minimizing effects due to the domestication of Chinese chestnut trees. We collected fresh leaves from the first year branches, and used silica gel dried leaves for DNA-seq to produce genomic sequences of population samples for each Castanea tree. We also collected fresh leaves from eight Castanopsis trees and used them as the outgroup in the analyses of population genomics described below. For each chestnut tree, genomic DNA was extracted using a standard protocol. We re-sequenced the individually indexed genomic libraries from all trees to an expected depth of $22 \times$ per tree using the Illumina Genome Analyzer (HiSeq 2500), with an insert size of $350 \mathrm{bp}$ and a read length of $150 \mathrm{bp}$ The raw reads so generated were subject to quality control using FASTX-Toolkit 
(http://hannonlab.cshl.edu/fastx_toolkit/). Bases with Phred quality score $\leq 20$ were defined as low quality. Low-quality bases were masked, and were trimmed if they were at end of the read. Reads with low-quality bases $>95 \%$ of read length or with a length of $<30 \mathrm{bp}$ were discarded and reads were also trimmed of any adapter and repetitive telomere sequences.

Mapping and calling. High-quality clean reads were mapped to the C. mollissima reference genome using BWA-MEM (0.7.16a-r1181) with default settings ${ }^{28}$. Then we used Picard-tools v1.92 (https://picard.sourceforge.net) to assign read group information containing library, lane, and sample identity, sort the SAM-format files and remove reads marked as duplicates, and generate BAM-format files. We used the HaplotypeCaller tool in Genome Analysis Toolkit (GATK) v4.0.8.131, to call genotypes per site and generated a GVCF format file for each tree. Then we used the GenotypeGVCFs and SelectVariants tools to obtain a list of potential SNPs for each tree. We used a hard-filtering approach to filter raw SNPs for each tree. We determined the filtering rule $\mathrm{DP}<8.0 \| \mathrm{DP}>60.0|| \mathrm{QD}<2.0||$ MQRankSum $<$

-12.5 || ReadPosRankSum $<-8.0$ || MQ $<40.0$ || FS $>60.0$ || SOR $>3.0$ by calculating the distribution of each statistic and used the VariantFiltration tool to perform the hard filtering. If an SNP site was within a distance $<3 \mathrm{bps}$ of an indel, it was marked as missing. For homozygous-reference calls, we further filtered by minimum and maximum depth ( $\mathrm{DP}<8.0$ and $\mathrm{DP}>60.0)$. Through these filtration steps we generated high-confidence SNPs and also invariant homozygous sites for each tree. The indels identified by the HaplotypeCaller tool, and other sites not satisfying the filtering criteria, were documented as missing. For each tree, the high-confidence SNPs and invariant sites were used to reconstruct FASTA format files.

Validation of SNP quality. Four trees of $C$. mollissima and three trees of $C$. henryi were used to assess the quality of the SNPs called by the GATK4-pipeline above, based on duplicated re-sequencing. For each of seven trees, two separate DNA samples were sequenced. These datasets were processed in a pipeline identical to that used for processing from quality control to the GATK4-pipeline, ignoring the fact that they were duplicates. Then we compared the genotypes of the two replicate samples for each of the seven trees. We also verified the SNPs using resequenced population data from C. mollissima and C. henryi. The rationale is that if a heterozygotic genotype from one tree genome cannot be detected in the sequencing data from the replicate or be found in population samples, it is treated as an unverified genotype.

Generating a 2D-SFS dataset. We used ANGSD v0. $928^{34}$ to generate beagleformat files based on filtered reads with a minimal mapping quality score of 30 and filtered bases with a minimal quality score of 20 in BAM-format files. The genomic sequences of eight Castanopsis trees, generated by GATK4 and custom python3 scripts, were used as outgroup. We used the -dosaf implementation to calculate the site allele frequency likelihood based on the samtool ${ }^{55}$ genotype likelihood model for all sites, and then employed the realSFS implementation to obtain a maximum likelihood estimate of the unfolded SFS using the Expectation Maximization (EM) algorithm ${ }^{56}$.

Individual clustering. We performed PCoA analysis using R, based on the variants detected by ANGSD-pipeline (with parameters -minMapQ 30 -minQ 20 -GL 2 -doMajorMinor 1 -doMaf 1 -SNP_pval 2e-6 -doIBS 1 -doCounts 1 -doCov 1 -makeMatrix 1 -minMaf 0.05). We ran ADMIXTURE ver. $1.23^{32}$ with crossvalidation for numbers of genetic clusters $(K)$ from 1 to 10 to infer the individual ancestry proportions with default settings. We used PLINK v1.0757 to reduce the LD effect. The optimum number of clusters $(K)$ was determined using the crossvalidation errors. Furthermore, we analyzed our dataset using another robust but time-consuming algorithm implemented in FASTSTRUCTURE ${ }^{33}$.

Population differentiation. We computed $D_{\mathrm{XY}}$ and $F_{\mathrm{ST}}$ for sliding windows (100 kbp nonoverlapping window) using custom python3 scripts, to examine the population differentiation between each pair of four taxa according to the equation proposed by Hudson, Slatkin and Maddison ${ }^{58-60}$. Furthermore, we defined a simple statistic $\max F_{\mathrm{ST}}$, which is the maximum $F_{\mathrm{ST}} / \mathrm{bp}$ in a predefined window $(100 \mathrm{~kb})$, to investigate the distribution of fixed differences along the genome. For each taxon, only SNPs with sample size $\geq 20$ were considered for computation.

The hhs test. We defined a statistic $h h s=d_{\mathrm{om}} / d_{\mathrm{hm}}$ in which $d_{\mathrm{om}}$ was the mean number of nucleotide differences between $C$. henryi var. omeiensis and C. mollissima and $d_{\mathrm{hm}}$ was the mean number of nucleotide differences between $C$. henryi var. henryi and C. mollissima. Given a genomic region with length of $l$, then the equation for $h h s$ can be written as

$$
h h s=\frac{\sum_{k=1}^{l} p_{o, k} q_{m, k}+p_{m, k} q_{o, k}}{\sum_{k=1}^{l} p_{h, k} q_{m, k}+p_{m, k} q_{h, k}}
$$

where $p_{x, k}$ and $q_{x, k}$ denote the frequencies of two alleles at site $k$ in taxon $x$. If the genetic compositions of $C$. henryi var. omeiensis were fully derived from $C$. henryi var. henryi, hhs would be equal to 1.0; if C. mollissima contributed any genetic information to $C$. henryi var. omeiensis, hhs would be $<1.0$ at some loci. We used the bootstrap method with 1000 replications to test whether $h h s=1.0$, applying the sliding-window approach with a window size of $100 \mathrm{kbp}$. For each replicate, we sampled windows randomly using the R function sample() and computed the $h$ hs value for each window. The $h$ hs value for each replicate was computed as the mean of these sampled windows. We then summarized $h h s$ values of all replicates.

Coalescent test. We employed fastsimcoal ${ }^{61,62}$, to estimate the $H$ parameter in a coalescent model based on the 2D-SFS format datasets. The $H$ parameter ${ }^{63}$ here represents the relative contribution from the C. mollissima genomes (Figs. 2 and S3.1.1). We removed the C. seguinii samples when testing for genomic mosaicism. We compared three models using the Akaike Information Criterion (AIC) method (Supplementary Fig. 3). In model $1(H=0)$, C. henryi var. omeiensis diverged from C. henryi var. henryi. In model $2(H=1), C$. henryi var. omeiensis diverged from $C$ mollissima. Model $3(0<H<1)$ represents a HHS model in which the genome of $C$. henryi var. omeiensis was derived from hybridization between C. henryi var. henryi and C. mollissima. We calculated likelihoods for each model based on 300,000 coalescent simulations. The likelihood and parameters were estimated in $50 \mathrm{ECM}$ cycles. The rate was set to $5 \times 10^{-9}$ substitutions per site per year ${ }^{64}$, and 15 years per generation was assumed. The $-\mathrm{C}$ parameter, that is the minimum size of entry of the observed and simulated SFS, was set to 5 . Standard deviations were determined from estimates from 20 duplicated computations with different seed numbers.

HyDe analyses. The $\mathrm{HyDe}^{35}$ program detects signal of hybridization between two non-sister lineages, using the unbiased statistic of the $H$ parameter proposed by Kubatko and Chifman ${ }^{37}$. We combined the $\mathrm{HyDe}^{35}$ program with a slidingwindow approach to estimate the $H$ parameter for each nonoverlapping window with sizes of $100 \mathrm{kbp}$ and $1 \mathrm{Mbp}$ across the genome. We used run hyde.py to examine whether genomic signal of hybridization could be detected in samples of C. henryi var. omeiensis based on the sliding-window approach (window sizes: 100 $\mathrm{kbp}$ and $1 \mathrm{Mbp}$ ). Then we used individual_hyde.py to estimate the $H$ parameter at an individual scale for each window (1 Mbp) and summarized all results to compute the $H$ parameter for each $C$. henryi var. omeiensis individual. For each of the 11 hybrids possibly produced by $C$. seguinii $\times$ C. mollissima, we also performed the HyDe analysis with C. seguinii and C. mollissima as parents.

PSMC and SMC ++ analyses. We applied the Pairwise Sequentially Markovian Coalescent (PSMC) model $^{65}$ to reconstruct demographic histories for C. mollissima and its relatives. We ran PSMC on each individual tree of the four taxa collected for this study based on the high-confidence sequences generated by our GATK4pipeline. We set the ratio of $\theta$ to $\rho$ (-r 2); the -g parameter (number of years per generation) to 15 ; the $-p$ parameter to $64 \times 2$; and the rate to $5 \times 10^{-9}$ substitutions per site per year ${ }^{64}$. We presented all results for each individual of the four lineages using one coordinate system to ensure that the results were reliable, using the program psmc_plot.pl from the PSMC package. To resolve the recent demographic histories clearly and robustly, we also inferred the demographic history using SMC++ (https://github.com/popgenmethods/smcpp). For each individual sample, we generated a whole-genome diploid consensus sequence with the highconfidence SNPs and invariant homozygous sites with the GATK4-pipeline. To test the effects of the $-\mathrm{d}$ parameter, we ran $\mathrm{SMC}++$ for each tree and the corresponding taxon. That is, each tree was set as a discrete sample and resequencing samples from each taxon were used in each SMC++-estimate analysis. We set the -spline parameter to the piecewise type; the -knots parameter to 40 and the -timepoints parameter from 1 to 5,000,000. Finally, we used SMC++-plot to summarize and depict the demographic histories of the four taxa.

Recombination map construction. We used LDhelmet v1.944 to generate a finescale recombination map for C. mollissima, based on the genomic sequences generated by the GATK4-pipeline. To infer recombination rates, the LDhelmet program requires: (1) the population mutation rate $(\theta),(2)$ phased haplotypes, $(3)$ the block penalty, (4) the ancestral allelic state per SNP used as prior, and (5) a mutation matrix for the focused species. Recent studies have shown that past bottleneck events may affect the estimation of recombination rates ${ }^{45}$, thus we took recent demographic changes in C. mollissima into account using LDpop ${ }^{45}$. LDhelmet and LDpop can only process up to 50 haplotypes, so we retained 25 trees of C. mollissima with long genome sequences (lower proportion of missing bases throughout the genome). We used the observed heterozygosity in 43 C. mollissima trees as the population mutation rate (here we used 0.0066 ). We used beagle $5^{66,67}$ to generate the phased haplotypes for variants in each of the assembled contigs. To reduce the effects of missing bases, we retained only those SNPs with no more than $20 \%$ missing bases. In two recent studies ${ }^{38,68}$, simulation-based analyses showed that LDhelmet performed better in simulations with a block penalty of 5 than with a higher block penalty, such as 50 . We used a block penalty of 5 in our analysis. We applied a parsimony-based method ${ }^{44}$ to infer the ancestral allele distribution at each site in C. mollissima by comparison with the aligned outgroup reference genomes of $C$. henryi var. henryi, var. omeiensis and C. seguinii. For each dimorphic site in C. mollissima genomes, if the alleles of the three outgroups were not all missing for this site and if they collectively exhibited precisely one of the four 
possible nucleotides, and if this allele agreed with one of the two observed in $C$. mollissima, then this was designated as the ancestral allelic state. Otherwise, it was considered to be unknown and we used the uniform distribution as a prior over the ancestral allele. To consider uncertainty in ancestral allele reconstruction, we set the prior for the putative ancestral allele as 0.91 and the priors for the other three bases as 0.03 . When estimating the mutation matrix for C. mollissima, we considered only biallelic SNPs. We then followed the Chan et al. ${ }^{44}$ approach to estimate the mutation matrix for this species (Supplementary Table 10). We used LDpop to generate the lookup table, which was used in LDhelmet. We computed a likelihood lookup table for a grid of $\rho$ values (-rh $0.0,0.01,1.0,1.0,100.0)$, for each set of haplotypes ( $-n 50)$, for each of 422 contigs. We set the -th parameters to 0.0066 and set the --approx option. The SMC++ results for $C$. mollissima were set in LDhelmet. Then we converted the format of the lookup table using convert_table from the LDhelmet package. We used the rjMCMC procedure with a burn-in of 100,000 and ran the Markov chain for 1 million iterations. After excluding SNPs for which recombination rate was estimated to be implausibly higher than $20 \times \theta$ (here we used 0.132), similar to the Schumer et al. ${ }^{38}$ settings, we finally summarized recombination rates in $100 \mathrm{~kb}$ windows (Fig. 3).

Correlation between $\boldsymbol{\rho}$ and $\boldsymbol{H}$. We examined the possible correlation between the recombination rate and genomic contribution from C. mollissima to $C$. henryi var. omeiensis $(H)$ using the $\mathrm{R}$ function $\operatorname{lm}()$. We also calculated the correlation between the recombination rate and genomic contribution from C. mollissima to C. seguinii.

Barrier genomic regions. We used $\mathrm{HyDe}^{35}$ and sliding-window analysis (100 kbp) to identify the windows that showed significant hybridization between $C$. seguinii and C. henryi var. henryi, with C. mollissima as the ingroup $(P<0.05)$. Then, with C. seguinii as the ingroup, we identified those loci that showed significant hybridization between C. mollissima and C. henryi var. henryi. For loci in $M_{\mathrm{hs}}$ and $M_{\mathrm{hm}}$, gene exchange between $C$. henryi and $\mathrm{A}_{\mathrm{sm}}$ could occur if migration rates were positive. We used the HyDe program and sliding-window analysis with a window size of $100 \mathrm{kbp}$ to perform local ancestry analysis; that is, to examine the source of genetic composition for each C. henryi var. omeiensis genomic window. For the test with the tree topology ((henryi, (omeiensis, mollissima)), outgroup) in a genomic window, if there was no significant signal of gene flow, this genomic window for $C$. henryi var. omeiensis was determined to be derived from C. mollissima. For the test with the tree topology ((mollissima, (omeiensis, henryi)), outgroup) in a genomic window, if there was no significant signal of gene flow, this genomic window for $C$. henryi var. omeiensis was determined to be sourced from $C$. henryi var. henryi.

Testing barrier regions. We used the coalescence-based method implemented in fastsimcoal $2^{61}$ to test whether the rate of migration between C. mollissima and $C$. henryi var. henryi $\left(M_{\mathrm{hm}}\right)$ was lower than the ancestral migration rate $\left(M_{\mathrm{anc}}\right)$ before time $t_{2}$ in the candidate barrier genomic windows (Supplementary Fig. 7). Two models were designed. In model A, $M_{\mathrm{hm}}<M_{\mathrm{anc}}$; and in model B, $M_{\mathrm{hm}} \geq M_{\mathrm{anc}}$ or $M_{\mathrm{hm}}=M_{\mathrm{anc}}$. We used the ANGSD-pipeline to generate the unfolded 2D-SFS format datasets for C. mollissima and C. henryi var. henryi in candidate barrier genomic windows. In this coalescent analysis using fastsimcoal2, we calculated the likelihood of each model based on 300,000 coalescent simulations. The likelihood and parameters were estimated in 50 ECM cycles. The -C parameter was set to 5 and the AIC values were used to compare the two models. For robustness, we performed 20 duplicated tests with different seed numbers for the dataset generated from the 88 candidate barrier genomic windows.

Relationship between barriers and $\rho$. We analyzed the relationship between $\rho$ and the number of barrier windows sourced from C. henryi var. henryi within the genome of hybrid lineage using the $\mathrm{R}$ function $\operatorname{lm}()$. The barrier windows from the major parent were set to be 1 and the other windows to be 0 .

Annotation of barrier regions. We annotated the functions of genes in the candidate barrier genomic regions, using the FLOR-ID database ${ }^{47}$. The genes that passed an $E$-value filtration $(1 \mathrm{e}-10)$ were further manually checked through comparison of their top blast hits in the FLOR-ID and SwissProt databases.

Reporting summary. Further information on research design is available in the Nature Research Reporting Summary linked to this article.

\section{Data availability}

Data supporting the findings of this work are available within the paper and its Supplementary Information files. A reporting summary for this article is available as a Supplementary Information file. The datasets generated and analyzed during the current study are available from the corresponding author upon request. All raw sequence reads have been deposited in the NCBI SRA database under BioProject accession number PRJNA540917 [https://www.ncbi.nlm.nih.gov/bioproject/540917]. Assembly and annotation of Castanea mollissima genome are available at GitHub [https://github.com/ yongshuai-sun/hhs-omei]. The source data underlying Figs. 1b-g, 2b,c, and 3, as well as
Supplementary Figs. 2 and 5-7 are provided as a Source data file. Source data file is also available at GitHub [https://github.com/yongshuai-sun/hhs-omei].

\section{Code availability}

The scripts used in this study are available at GitHub [https://github.com/yongshuai-sun/ hhs-omei].

Received: 26 November 2019; Accepted: 9 June 2020;

Published online: 06 July 2020

\section{References}

1. Abbott, R. J. et al. Hybridization and speciation. J. Evol. Biol. 26, 229-246 (2013).

2. Mallet, J. Hybrid speciation. Nature 446, 279-283 (2007).

3. Schumer, M., Cui, R., Rosenthal, G. G. \& Andolfatto, P. Reproductive isolation of hybrid populations driven by genetic incompatibilities. PLoS Genet. 11, e1005041 (2015).

4. Hoffmann, A. A. \& Rieseberg, L. H. Revisiting the impact of inversions in evolution: from population genetic markers to drivers of adaptive shifts and speciation? Annu. Rev. Ecol. Evol. Syst. 39, 21-42 (2008).

5. Buerkle, C. A., Morris, R. J., Asmussen, M. A. \& Rieseberg, L. H. The likelihood of homoploid hybrid speciation. Heredity 84, 441-451 (2000).

6. Marques, D. A., Meier, J. I. \& Seehausen, O. A combinatorial view on speciation and adaptive radiation. Trends Ecol. Evol. 34, 531-544 (2019).

7. Brennan, A. C., Hiscock, S. J. \& Abbott, R. J. Completing the hybridization triangle: the inheritance of genetic incompatibilities during homoploid hybrid speciation in ragworts (Senecio). AoB Plants 11, ply078 (2019).

8. Hermansen, J. S. et al. Hybrid speciation through sorting of parental incompatibilities in Italian sparrows. Mol. Ecol. 23, 5831-5842 (2015).

9. Rieseberg, L. H. et al. Major ecological transitions in wild sunflowers facilitated by hybridization. Science 301, 1211-1216 (2003).

10. Schumer, M., Rosenthal, G. G. \& Andolfatto, P. How common is homoploid hybrid speciation? Evolution 68, 1553-1560 (2014).

11. Baack, E., Melo, M. C., Rieseberg, L. H. \& Ortiz-Barrientos, D. The origins of reproductive isolation in plants. New Phytol. 207, 968-984 (2015).

12. Ravinet, $M$. et al. Interpreting the genomic landscape of speciation: a road map for finding barriers to gene flow. J. Evol. Biol. 30, 1450 (2017).

13. Gompert, Z. \& Buerkle, A. Bayesian estimation of genomic clines. Mol. Ecol. 20, 2111-2127 (2011)

14. Schumer, M. et al. High-resolution mapping reveals hundreds of genetic incompatibilities in hybridizing fish species. eLife 3, e02535 (2014).

15. Rigling, D. \& Prospero, S. Cryphonectria parasitica, the causal agent of chestnut blight: Invasion history, population biology and disease control. Mol. Plant Pathol. 19, 7-20 (2018).

16. Kuhlman, E. G. Chestnut Blight. Science 210, 1199-1200 (1980).

17. Thompson, H. Plant science: the chestnut resurrection. Nature 490, 22-23 (2012).

18. Gabriel, Popkin Can a transgenic chestnut restore a forest icon? Science 361, 830-831 (2018).

19. Huang, C., Zhang, Y. \& Bruce, B. Fagaceae in Flora of China, Vol. 4 (eds Wu, Z. Y. \& Peter, R. H.) 314-400 (Science Press, 1999).

20. Liu, G. \& Fang, J. Spatial patterns of chestnut (Castanea millissima) and its species geographical distribution in China. Acta Ecol. Sin. 21, 164-170 (2001).

21. Liu, G. B. et al. Study on the distant hybridization compatibility between Castanea mollissima and C. henryi. New Hortic. 15, 1-4 (2013).

22. Lang, P., Dane, F., Kubisiak, T. L. \& Huang, H. Molecular evidence for an Asian origin and a unique westward migration of species in the genus Castanea via Europe to North America. Mol. Phylogenet. Evol. 43, 49-59 (2007).

23. Lang, P. \& Huang, H. Genetic diversity and geographic variation in natural populations of the endemic Castanea species in China. Acta Bot. Sin. 41, 651-657 (1999).

24. Clarke, J. et al. Continuous base identification for single-molecule nanopore DNA sequencing. Nat. Biotechnol. 4, 265 (2009).

25. Lieberman-Aiden, E. et al. Comprehensive mapping of long-range interactions reveals folding principles of the human genome. Science 326, 289-293 (2009).

26. Xing, Y. et al. Hybrid de novo genome assembly of Chinese chestnut (Castanea mollissima). GigaScience 8, giz112 (2019).

27. Marçais, G. \& Kingsford, C. A fast, lock-free approach for efficient parallel counting of occurrences of k-mers. Bioinformatics 27, 764 (2011).

28. Li, H. Aligning Sequence Reads, Clone Sequences and Assembly Contigs with BWA-MEM. https://arxiv.org/abs/1303.3997v2 (2013).

29. Waterhouse, R. M. et al. BUSCO applications from quality assessments to gene prediction and phylogenomics. Mol. Biol. Evol. 35, 543-548 (2017). 
30. Staton, M. et al. The Chinese Chestnut Genome: A Reference for Species Restoration. https://www.biorxiv.org/content/10.1101/615047v1, 615047 (2019).

31. Depristo, M. A. et al. A framework for variation discovery and genotyping using next-generation DNA sequencing data. Nat. Genet. 43, 491-498 (2011).

32. Alexander, D. H. \& Lange, K. Enhancements to the ADMIXTURE algorithm for individual ancestry estimation. BMC Bioinforma. 12, 246 (2011).

33. Raj, A., Stephens, M. \& PritchardJ. K. fastSTRUCTURE: variational inference of population structure in large SNP data sets. Genetics 197, 573-589 (2014).

34. Korneliussen, T. S., Albrechtsen, A. \& Nielsen, R. ANGSD: analysis of next generation sequencing data. BMC Bioinforma. 15, 356 (2014).

35. Blischak, P. D., Chifman, J., Wolfe, A. D. \& Kubatko, L. S. HyDe: a python package for genome-scale hybridization detection. Syst. Biol. 67, 821-829 (2018).

36. Martin, S. H., Davey, J. W. \& Jiggins, C. D. Evaluating the use of ABBA-BABA statistics to locate introgressed loci. Mol. Biol. Evol. 32, 244-257, https://doi. org/10.1093/molbev/msu269 (2015).

37. Kubatko, L. S. \& Chifman, J. An invariants-based method for efficient identification of hybrid species from large-scale genomic data. BMC Evol. Biol. 19, 112-124 (2019).

38. Schumer, M. et al. Natural selection interacts with recombination to shape the evolution of hybrid genomes. Science 360, 656-660 (2018).

39. Yeaman, S. \& Whitlock, M. C. The genetic architecture of adaptation under migration-selection balance. Evolution 65, 1897-1911 (2011).

40. Akerman, A. \& Bürger, R. The consequences of gene flow for local adaptation and differentiation: a two-locus two-deme model. J. Math. Biol. 68, 1135-1198 (2014).

41. Kim, B. Y., Huber, C. D. \& Lohmueller, K. E. Deleterious variation shapes the genomic landscape of introgression. PLoS Genet. 14, el007741 (2018).

42. Lamichhaney, S. et al. Rapid hybrid speciation in Darwin's finches. Science 359, 224-228 (2018).

43. Aeschbacher, S., Selby, J. P., Willis, J. H. \& Coop, G. Population-genomic inference of the strength and timing of selection against gene flow. PNAS 114, 7061-7066 (2017).

44. Chan, A. H., Jenkins, P. A. \& Song, Y. S. Genome-wide fine-scale recombination rate variation in Drosophila melanogaster. PLoS Genet. 8, e1003090 (2012).

45. Kamm, J. A., Spence, J. P., Chan, J. \& Song, Y. S. Two-locus likelihoods under variable population size and fine-scale recombination rate estimation. Genetics 203, 1381 (2016).

46. Martin, S., Davey, J., Salazar, C. \& Jiggins, C. Recombination rate variation shapes barriers to introgression across butterfly genomes. PLoS Biol. 17, e2006288 (2019).

47. Bouché, F., Lobet, G., Tocquin, P. \& Périlleux, C. FLOR-ID: an interactive database of flowering-time gene networks in Arabidopsis thaliana. Nucleic Acids Res. 44, D1167-D1171 (2016).

48. Jin, J. B. et al. The SUMO E3 ligase, AtSIZ1, regulates flowering by controlling a salicylic acid-mediated floral promotion pathway and through affects on FLC chromatin structure. Plant J. 53, 530-540 (2008).

49. Lu, F., Cui, X., Zhang, S., Liu, C. \& Cao, X. JMJ14 is an H3K4 demethylase regulating flowering time in Arabidopsis. Cell Res. 20, 387-390 (2010).

50. Jesús, M. et al. Speciation by hybridization in Heliconius butterflies. Nature 441, 868-871 (2006)

51. Berner, D. \& Roesti, M. Genomics of adaptive divergence with chromosomescale heterogeneity in crossover rate. Mol. Ecol. 26, 6351-6369 (2017).

52. Yeaman, S. \& Whitlock, M. C. The genetic architecture of adaptation under migration-selection balance. Evolution 65, 1897-1911 (2011).

53. Jones, P. et al. InterProScan 5: genome-scale protein function classification. Bioinformatics 30, 1236-1240 (2014).

54. Moriya, Y., Itoh, M., Okuda, S., Yoshizawa, A. C. \& Kanehisa, M. KAAS: an automatic genome annotation and pathway reconstruction server. Nucleic Acids Res. 35, W182-W185 (2007).

55. Li, H., Handsaker, B., Wysoker, A., Fennell, T. \& Ruan, J. The sequence alignment-map format and SAMtools. Bioinformatics 25, 2078-2079 (2009).

56. Kim, S. et al. Estimation of allele frequency and association mapping using next-generation sequencing data. BMC Bioinforma. 12, 231 (2011).

57. Purcell, S. et al. PLINK: a tool set for whole-genome association and population-based linkage analyses. Am. J. Hum. Genet. 81, 559-575 (2007).

58. Hudson, R. R., Slatkin, M. \& Maddison, W. P. Estimation of levels of gene flow from DNA sequence data. Genetics 132, 583-589 (1992).

59. Bhatia, G., Patterson, N., Sankararaman, S. \& Price, A. L. Estimating and interpreting FST: the impact of rare variants. Genome Res. 23, 1514-1521 (2013).
60. Wakeley, J. The variance of pairwise nucleotide differences in two populations with migration. Theor. Popul. Biol. 49, 39 (1996).

61. Excoffier, L., Dupanloup, I., Huerta-Sánchez, E., Sousa, V. C. \& Foll, M. Robust demographic inference from genomic and SNP data. PLoS Genet. 9 , e1003905 (2013).

62. Excoffier, L. \& Foll, M. Fastsimcoal: a continuous-time coalescent simulator of genomic diversity under arbitrarily complex evolutionary scenarios. Bioinformatics 27, 1332-1334 (2011).

63. Sun, Y. et al. Evolutionary history of Purple cone spruce (Picea purpurea) in the Qinghai-Tibet Plateau: homoploid hybrid origin and Pleistocene expansion. Mol. Ecol. 23, 343-359 (2014).

64. De La Torre, A. R., Li, Z., Van de Peer, Y. \& Ingvarsson, P. K. Contrasting rates of molecular evolution and patterns of selection among gymnosperms and flowering plants. Mol. Biol. Evol. 34, 1363-1377 (2017).

65. Heng, L. \& Richard, D. Inference of human population history from individual whole-genome sequences. Nature 475, 493 (2011).

66. Browning, S. R. \& Browning, B. L. Rapid and accurate haplotype phasing and missing-data inference for whole-genome association studies by use of localized haplotype clustering. Am. J. Hum. Genet. 81, 1084-1097 (2007).

67. Browning, B. L., Zhou, Y. \& Browning, S. R. A one-penny imputed genome from next generation reference panels. Am. J. Hum. Genet. 103, 338-348 (2018).

68. Sonal, S. et al. Stable recombination hotspots in birds. Science 350, 928-932 (2015).

\section{Acknowledgements}

This research was supported by the National Natural Science Foundation of China (gran numbers 31670665), Yunling Youth Talents Plan of Yunnan Province, Xishuangbanna Tropical Botanical Garden, Youth Innovation Promotion Association CAS and CAS Light of West China Program.

\section{Author contributions}

Y.S. conceived the project and managed financial support and genome sequencing. Z.L., X.Z., and Y.S. collected data. Y.S. and H.M. carried out data analyses. Y.S. proposed the $\max F_{\mathrm{ST}}$ and $h h s$ methods. Y.S. wrote the paper.

\section{Competing interests}

The authors declare no competing interests.

\section{Additional information}

Supplementary information is available for this paper at https://doi.org/10.1038/s41467 020-17111-w.

Correspondence and requests for materials should be addressed to Y.S.

Peer review information Nature Communications thanks the anonymous reviewers for their contribution to the peer review of this work.

Reprints and permission information is available at http://www.nature.com/reprints

Publisher's note Springer Nature remains neutral with regard to jurisdictional claims in published maps and institutional affiliations.

Open Access This article is licensed under a Creative Commons Attribution 4.0 International License, which permits use, sharing, adaptation, distribution and reproduction in any medium or format, as long as you give appropriate credit to the original author(s) and the source, provide a link to the Creative Commons license, and indicate if changes were made. The images or other third party material in this article are included in the article's Creative Commons license, unless indicated otherwise in a credit line to the material. If material is not included in the article's Creative Commons license and your intended use is not permitted by statutory regulation or exceeds the permitted use, you will need to obtain permission directly from the copyright holder. To view a copy of this license, visit http://creativecommons.org/ licenses/by/4.0/

(C) The Author(s) 2020 